\title{
Indirect Treatment Comparison of the Efficacy and Safety of Sarilumab Monotherapy in Rheumatoid Arthritis Patients with Inadequate Response to Conventional Disease-Modifying Antirheumatic Drugs
}

\author{
Ernest Choy $\cdot$ Nick Freemantle $\cdot$ Clare Proudfoot $\cdot$ Chieh-I Chen • \\ Laurence Pollissard · Andreas Kuznik · Hubert van Hoogstraten • \\ Erin Mangan · Paulo Carita · Thi-Minh-Thao Huynh \\ Received: December 20, 2018 / Published online: March 12, 2019 \\ (C) The Author(s) 2019
}

\begin{abstract}
Introduction: To evaluate the comparative efficacy and safety of subcutaneous sarilumab $200 \mathrm{mg}$ monotherapy administered every 2 weeks (q2w) versus other monotherapies of biologic, targeted and conventional synthetic disease-modifying antirheumatic drugs (bDMARDs, tsDMARDs, csDMARDs) at recommended doses for treatment of rheumatoid
\end{abstract}

Enhanced Digital Features To view enhanced digital features for this article go to: https://doi.org/10.6084/ m9.figshare.7712498.

Electronic supplementary material The online version of this article (https://doi.org/10.1007/s12325019-00912-x) contains supplementary material, which is available to authorized users.

E. Choy

Cardiff University, Cardiff, UK

N. Freemantle

University College London, London, UK

C. Proudfoot

Formerly of Sanofi, Guildford, UK

C.-I. Chen · A. Kuznik · E. Mangan

Regeneron Pharmaceuticals, Inc., Tarrytown, NY, USA

L. Pollissard · P. Carita · Thi-Minh-ThaoHuynh ( $₫)$

Sanofi France, Chilly-Mazarin, France

e-mail: Thi-Minh-Thao.Huynh@sanofi.com

H. van Hoogstraten

Sanofi, Bridgewater, NJ, USA arthritis in patients who are intolerant of or inadequate responders to csDMARDs (csDMARD-IR).

Methods: A systematic literature review and network meta-analysis (NMA) were conducted on 24-week efficacy outcomes: Health Assessment Questionnaire Disability Index (HAQ-DI) score, American College of Rheumatology (ACR) 20/50/70 criteria, and European League Against Rheumatism Disease Activity Score 28-joint count erythrocyte sedimentation rate (DAS28) < 2.6. In addition, serious infections and serious adverse events (SI/SAE) were examined at 24 weeks.

Results: Nine trials were selected for the NMA. Sarilumab $200 \mathrm{mg}$ showed superiority versus adalimumab monotherapy on all efficacy outcomes and versus tofacitinib monotherapy on ACR20. Compared with csDMARDs, sarilumab $200 \mathrm{mg}$ showed superiority on ACR 20/50/70 criteria and DAS28 $<2.6$ but had similar efficacy on HAQ-DI. Efficacy of sarilumab $200 \mathrm{mg}$ was similar versus certolizumab, etanercept, tofacitinib and tocilizumab $8 \mathrm{mg} / \mathrm{kg}$ monotherapy across all efficacy outcomes. SI/SAE appeared similar for sarilumab $200 \mathrm{mg}$ versus all comparators.

Conclusion: In csDMARD-IR patients, sarilumab $200 \mathrm{mg}$ monotherapy has superior efficacy and similar safety versus csDMARDs, superior efficacy and similar safety versus adalimumab, and similar efficacy and safety versus bDMARDs and tsDMARDs. 
Funding: Sanofi and Regeneron Pharmaceuticals, Inc.

Keywords: Biologic disease-modifying antirheumatic drugs; Network meta-analysis; Rheumatoid arthritis; Rheumatology; Sarilumab monotherapy

\section{INTRODUCTION}

Sarilumab, a human monoclonal antibody, which blocks both the soluble and membrane forms of the interleukin-6 receptor, is a biologic disease-modifying antirheumatic drug (bDMARD) for use as monotherapy or in combination with conventional synthetic (cs) DMARDs for the treatment of adults with moderate-to-severe rheumatoid arthritis (RA) in patients who are intolerant or inadequate responders to csDMARDs (csDMARD-IR) [1-4]. Evaluation of the comparative effectiveness and safety of sarilumab against other DMARDs is needed to guide evidence-based medicine [5]; however, there are limited head-to-head data comparing sarilumab with other relevant RA treatments. In the absence of direct evidence, a network meta-analysis (NMA) facilitates evaluation of sarilumab against other treatments using a combination of direct and indirect trial data. This NMA was conducted to evaluate the comparative efficacy and safety of subcutaneous (SC) sarilumab $200 \mathrm{mg}$ monotherapy, administered every 2 weeks ( $(2 \mathrm{w})$ versus other globally approved monotherapies for RA. Comparator treatments included bDMARDs, targeted synthetic DMARDs (tsDMARDs) and csDMARDs at their recommended doses for the treatment of RA as monotherapy for csDMARD-IR. Comparative evidence of sarilumab in combination with csDMARDs is published elsewhere [6].

\section{METHODS}

A systematic literature review (SLR) and NMA were conducted following methods in line with Preferred Reporting Items for Systematic Reviews and Meta-Analyses (PRISMA) guidelines [7] and recommended in the current National Institute for Health and Care Excellence (NICE) specification for manufacturer and sponsor submission of evidence [8], as well as the 2016 NICE technology appraisal of adalimumab, etanercept, infliximab, certolizumab pegol, golimumab, tocilizumab, and abatacept for RA [9]. Due to the nature of the study it was not registered with clinicaltrials.gov or a similar body. This article is based on previously conducted studies and does not contain any studies with human participants or animals performed by any of the authors.

\section{Study Selection}

Searches for the SLR were conducted in the MEDLINE, EMBASE, and Cochrane databases (all without any time limit), plus conference proceedings since 2013 for evidence published until December 6, 2016. Studies were selected according to pre-defined population/intervention/comparator/outcome/study design criteria (Table 1) $[7,8,10,11]$. All titles, abstracts and articles were then screened independently by two researchers, with study selection following published best practice guidelines for indirect treatment comparisons $[8,10,11]$.

Data on study design, patient characteristics, efficacy, safety and patient-reported outcomes at the time points $12( \pm 4), 24( \pm 4)$ and $52( \pm 8)$ weeks for all studies (except open-label extensions) were extracted independently by two reviewers in a pre-defined data extraction process. Evidence for the NMA was filtered for drugs licensed for RA at doses approved in Europe, the USA and Canada. All trials comparing one intervention of interest with at least one other intervention of interest or methotrexate or $\geq 1$ csDMARD(s) were considered in the evidence base.

Small studies have been shown to distort meta-analyses [12] therefore, studies with fewer than 30 patients per arm were excluded. Studies which did not report any outcomes of interest were also excluded.

\section{Treatment Categorisation}

Different licensed dosages and different routes of administration [e.g., intravenous (IV) versus SC delivery)] of the same treatment were pooled in 
Table 1 Population/intervention/comparator/outcome/study design and search criteria for the SLR

\begin{tabular}{|c|c|c|c|}
\hline Criteria & \multicolumn{3}{|l|}{ Inclusion } \\
\hline Study design & \multicolumn{3}{|c|}{ Randomized controlled trials above phase I (including crossover studies up to time of crossover) } \\
\hline \multirow[t]{2}{*}{ Population } & \multirow{2}{*}{\multicolumn{3}{|c|}{$\begin{array}{l}\text { Adult patients (aged } \geq 18 \text { years) with moderately-to-severely active RA who have had inadequate } \\
\text { response to } \geq 1 \text { csDMARDs } \\
\text { Adult patients (aged } \geq 18 \text { years) with moderately-to-severely active RA who have had inadequate } \\
\text { response to } \geq 1 \mathrm{TNF} \alpha \text {-inhibitors }\end{array}$}} \\
\hline & & & \\
\hline \multirow{10}{*}{$\begin{array}{l}\text { Treatment/ } \\
\text { intervention }\end{array}$} & \multicolumn{3}{|c|}{ Interventions of interest (at any dosage or administration type $)^{a}$ : } \\
\hline & \multirow{2}{*}{$\begin{array}{l}\text { Sarilumab (REGN88, } \\
\text { SAR153191) }\end{array}$} & \multirow{2}{*}{$\begin{array}{l}\text { Tocilizumab (RoActemra/ } \\
\text { Actemra) }\end{array}$} & SB2 (Samsung Bioepis) \\
\hline & & & Inflectra (CT-P13) \\
\hline & Adalimumab (Humira) & Tofacitinib (Xeljanz) & Flixabi (Biogen) \\
\hline & Certolizumab (Cimzia) & SB4 (Samsung Bioepis) & Rituxan (GP2013) \\
\hline & Etanercept (Enbrel) & GP2015 (Sandoz) & Baricitinib (LY3009104, \\
\hline & Golimumab (Simponi) & ABP501 (Amgen) & INCB028050) \\
\hline & Infliximab (Remicade) & BI695501 (Boehringer) & \\
\hline & Abatacept (Orencia) & SB5 (Samsung Bioepis) & \\
\hline & $\begin{array}{l}\text { Rituximab (MabThera/ } \\
\text { Rituxan) }\end{array}$ & Remsima (CT-P13) & \\
\hline Comparator & \multicolumn{3}{|c|}{ Placebo or any of the above listed treatments as monotherapy } \\
\hline Outcomes & \multicolumn{3}{|c|}{ Efficacy, safety and patient reported outcomes at 24 weeks ( \pm 4 weeks) and 52 weeks ( \pm 8 weeks) } \\
\hline Time & \multicolumn{3}{|l|}{ No limit } \\
\hline Language & \multicolumn{3}{|l|}{ English language } \\
\hline
\end{tabular}

cs Conventional synthetic, DMARD disease-modifying antirheumatic drug, $R A$ rheumatoid arthritis, $T N F$ tumor necrosis factor

a Only interventions with global regulatory approval were included

many cases, on the basis of evidence of equivalence (Supplementary Table S1). These decisions were explored by examining forest plots of the odds ratio (OR) for American College of Rheumatology (ACR) 20\% response criteria (ACR20) at 24 weeks in individual studies by group of interventions. If the confidence intervals were overlapping (e.g., as for infliximab studies), the doses were pooled. The validity of the decisions was also confirmed via clinician input.

\section{Outcomes Examined}

Key efficacy endpoints were extracted and analyzed including ACR20, ACR 50\% response criteria (ACR50), ACR 70\% response criteria (ACR70), and the Health Assessment Questionnaire Disability Index (HAQ-DI) change from baseline. The European League Against Rheumatism (EULAR) Disease Activity Score 28-joint count (DAS28) remission (defined as DAS28 erythrocyte sedimentation rate or C-reactive protein $<2.6$ ) was also extracted; however, this endpoint was not analyzed given that the EULAR networks were small and a high level of variability was observed in response rates between the different studies. Safety endpoints included the proportion of patients with any serious infection (SI) and the proportion of patients with any serious adverse event (SAE). 
All efficacy and safety outcomes were examined at 24 weeks as this was the assessment period with the most data available for analysis.

\section{Network Meta-Analysis}

\section{Feasibility Assessment}

Prior to the conduct of the NMA, a feasibility assessment was conducted to assess the sufficiency of the evidence base to draw feasible networks for all outcomes of interest. The exchangeability assumption is critical and requires that selected trials measure the same underlying relative treatment effects. Deviations to this assumption can be evaluated through two metrics: heterogeneity (i.e., evaluation of comparability in characteristics and results across included studies) and consistency (i.e., evaluation of consistency between direct and indirect evidence). Effect modifiers were evaluated by establishing the link between patient characteristics at baseline and ACR20; only weight was identified as an effect modifier given the expected variation in patient characteristics across RA studies [11, 13, 14], which can limit the validity of indirect comparisons.

Variability of response in the placebo arms is an issue which can limit indirect comparisons, and the heterogeneity of RA studies has been previously noted [15] where the treatment effect expressed as log ORs has a negative relationship with the baseline risk $[9,16]$. While the common comparator across most monotherapy trials was an active comparator (adalimumab), for the few placebo-controlled studies, variability was therefore considered in the selection of models.

\section{Bayesian NMA}

The efficacy and safety of the treatments included in the analysis were evaluated using a Bayesian NMA approach [10, 14, 17], comprising a likelihood distribution, a model with parameters and prior distributions for these parameters. A linear model with normal likelihood distribution was used for continuous outcomes, and a binomial likelihood with a log link was used for the dichotomous outcomes $[15,18]$. Consistent with NICE guidelines, flat (non-informative) prior distributions were assumed for nearly all outcomes so as not to influence the observed results by the prior distribution [15]. Prior distributions of the baseline treatments and relative treatment effects were normal, with 0 mean and variance of 10,000 , with informative prior based on between study variance according to the recommendation of NICE in the case of limited data [15]. Randomand fixed-effects models were evaluated to allow for heterogeneity of treatment effects between studies, with the choice of base-case informed by Deviance Information Criterion (DIC) values and mean total residual deviance (compared against the number of fitted data points), as well as consistency with directly reported trial results [17]. Posterior densities for unknown parameters were estimated using Markov chain Monte Carlo simulations.

All results for OR-NMA and risk difference (RD)-NMA were based on 100,000 iterations on three chains, with a burn-in of 20,000 iterations. Convergence was assessed by visual inspection of trace plots. The accuracy of the posterior estimates was assessed using the Monte Carlo error for each parameter (Monte Carlo error $<1 \%$ of the posterior standard deviation). All models were implemented using WinBUGS. Results of the NMA are presented in terms of 'point estimates' (median of posterior) for the relative treatment effects, along with the $95 \%$ credible intervals.

\section{Scenario Analyses}

Two scenario analyses were conducted. The first excluded studies conducted in exclusively Asian populations (i.e., the SATORI, CHANGE and Etanercept 309 studies) to test the potential modifying effect of patient body weight (with Asian ethnicity serving as a proxy for populations with relatively lower body weight than other populations. For the second analysis, tumour necrosis factor (TNF)- $\alpha$ inhibitors were pooled together as a class. For the latter scenario analysis, ACR outcomes were compared with the base case which evaluated the TNF- $\alpha$ inhibitors individually. This scenario was evaluated to inform cost-effectiveness evaluations of sarilumab. 


\section{RESULTS}

\section{SLR Search and Selection}

Following the SLR search a total of 31 citations which met the screening criteria were retrieved (Fig. 1); these reported the results of 19 randomized clinical trials (RCTs). Five RCTs were excluded because the comparator was out of scope. Of the 14 RCTs which were included in the NMA feasibility assessment, 5 of these were excluded due to no outcomes of interest. A net of 9 RCTs were included in the NMA (Fig. 1), including the MONARCH trial of sarilumab versus adalimumab monotherapy (NCT02332590) [2].

\section{Network Meta-Analysis Evidence Base}

Evaluation of the evidence base indicated that the ACR networks, and in particular ACR20 (Fig. 2), were the most robust networks where

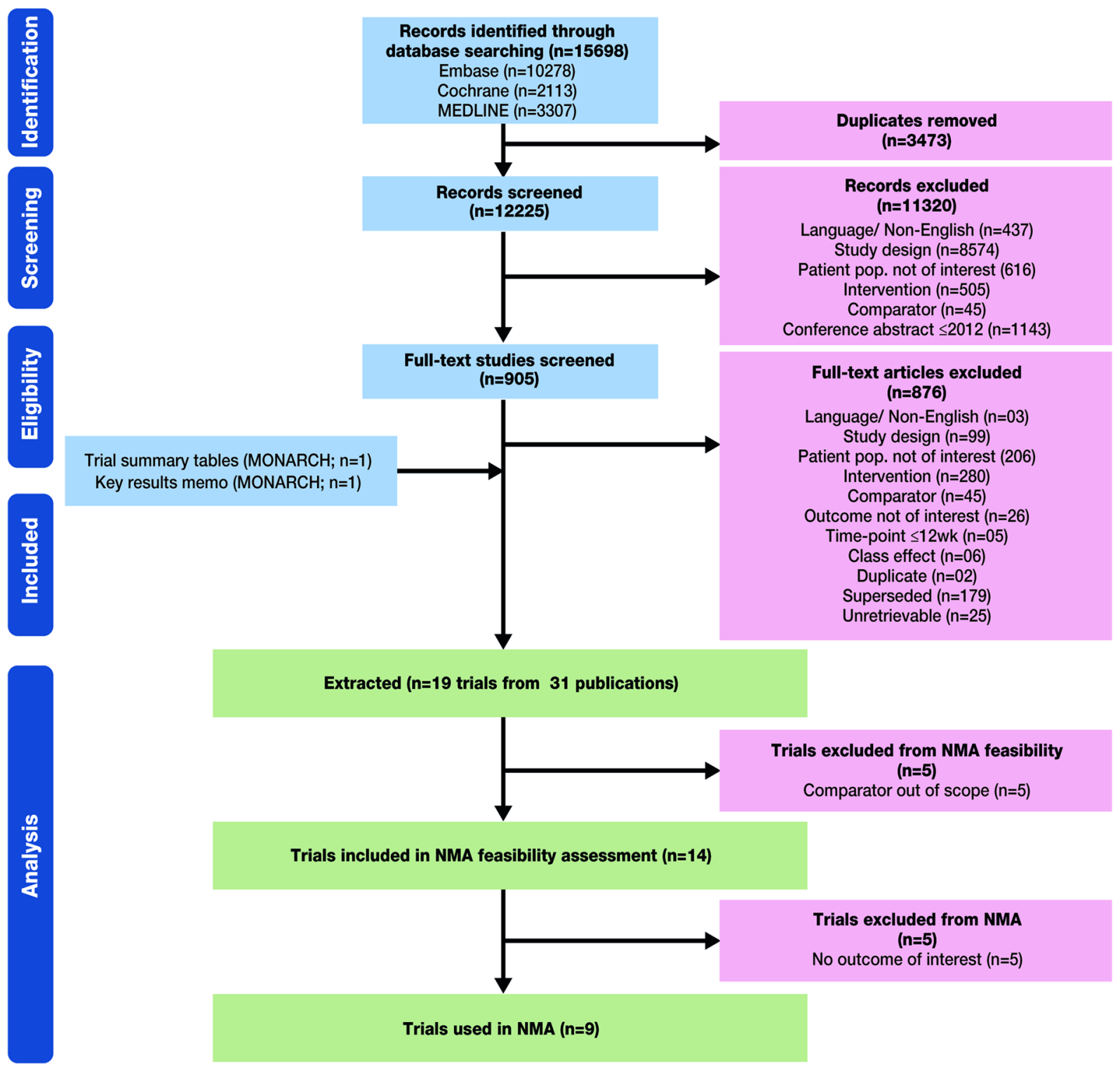

Fig. 1 Systematic review and network meta-analyses study selection flow chart. NMA Network meta-analysis 


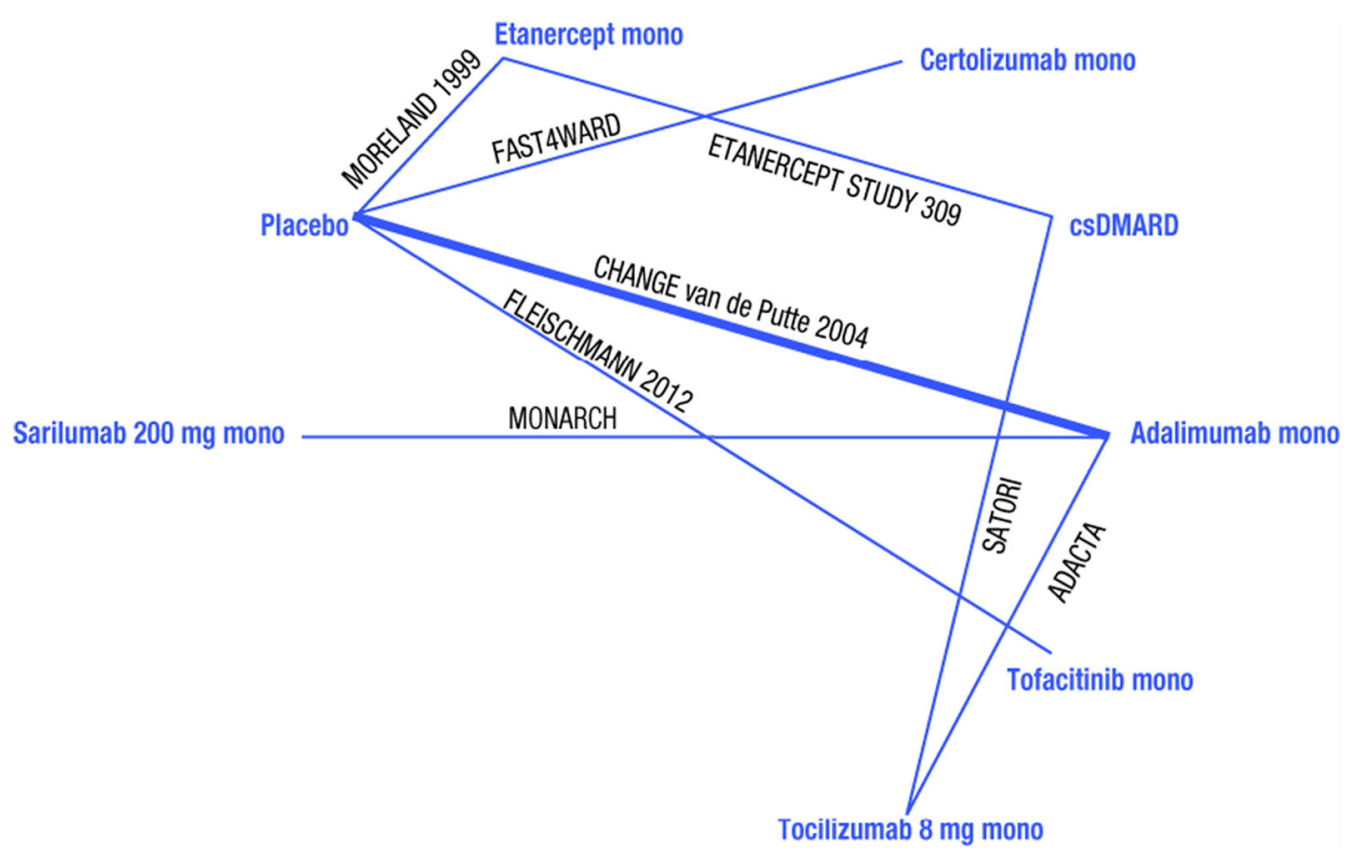

Fig. 2 Evidence base networks for American College of Rheumatology 20\% response rate outcomes at 24 weeks. csDMARD conventional synthetic disease-modifying antirheumatic drug

most interventions included were from multiple trials.

Key features of patient demographics and baseline data from the selected studies are provided in Supplementary Table S2. Among 9 trials included, 4 were phase III trials, 1 each was a phase II trial, phase II/III trial and phase IV trial; the trial phase was not mentioned for the remaining 2 trials. Study durations varied from 24 weeks up to 52 weeks; however, 1 doseranging study of tofacitinib compared with adalimumab was designed for cross-over to tofacitinib at week 12 for patients in the adalimumab arm [19]. Therefore, this study was included only for evaluating outcomes of tofacitinib versus placebo and included in the relevant networks. In 3 studies, patients had to have been on stable methotrexate for at least 12 weeks prior to entering the study; in another 3 studies this criterion was not required, and in the remaining studies no such information was reported.

Sample sizes varied from fewer than 50 patients to more than 150 patients per randomized group. Rescue medication was permitted in 5 of the trials and not reported in the remainder of the trials.

\section{Base Case Model Choice}

The feasibility assessment indicated that there was considerably less variability where the common comparator was an active comparator (adalimumab), with some degree of variability where placebo was the control arm. Therefore, a fixed-effects model using conventional OR model was selected as the base-case model for ACR20/50/70 outcomes (ACR20: DIC $=141.98$; ACR50: $\quad$ DIC $=135.62 ; \quad$ ACR70: $\quad$ IIC $=99.93)$. For the continuous efficacy outcome, HAQ-DI, a standard change from baseline NMA was conducted. In addition, the RD-NMA was applied for DAS28 $<2.6$ and safety outcomes due to convergence issues in the OR model and/or due to the relative rarity of the event.

\section{Base Case Network Meta-Analysis Results}

In the base case, NMA indicated superior efficacy for sarilumab $200 \mathrm{mg}$ versus adalimumab monotherapy on all efficacy outcomes and 


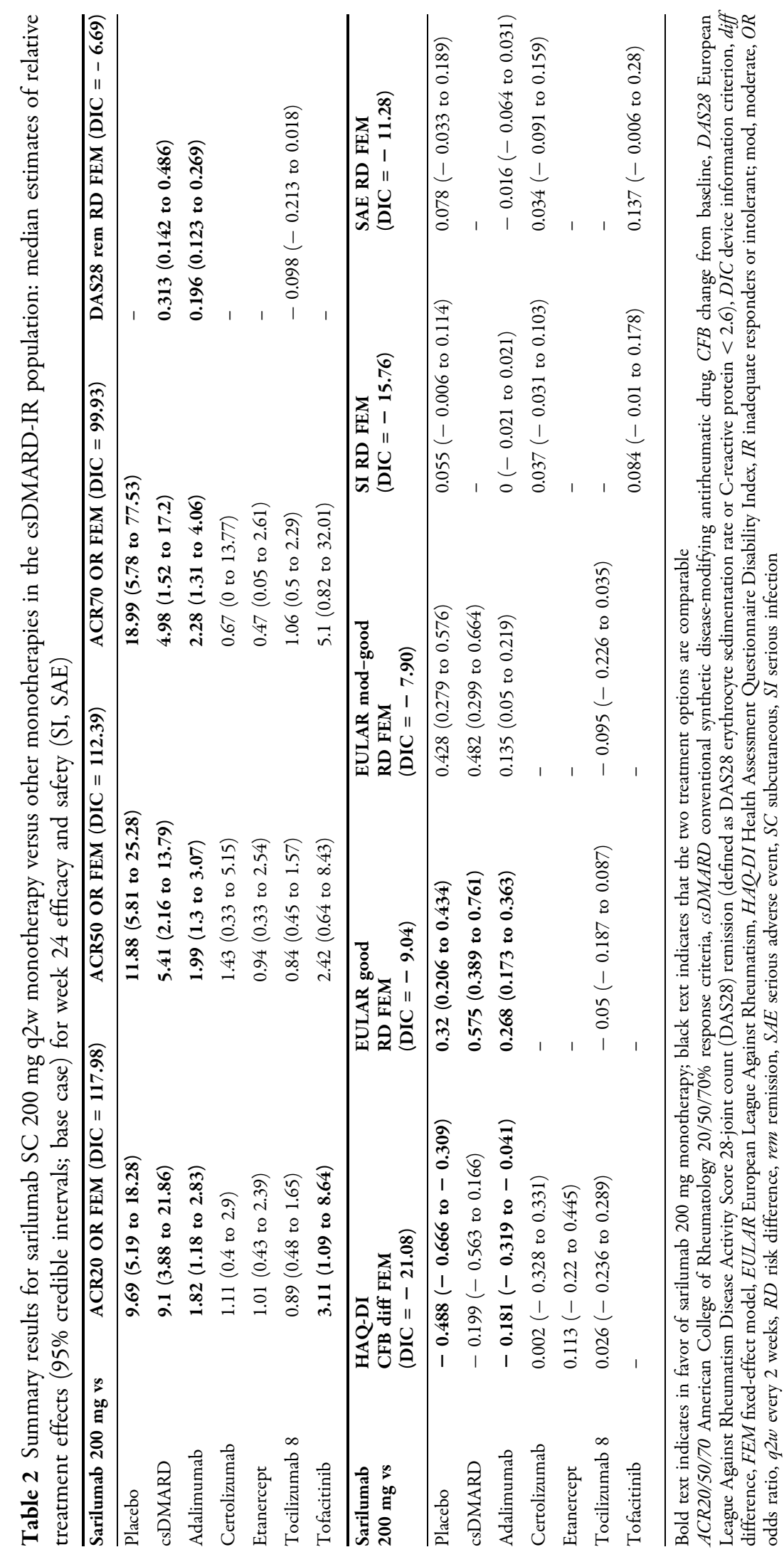


versus tofacitinib monotherapy on ACR20 (Table 2). Compared with csDMARDs, sarilumab $200 \mathrm{mg}$ had superior efficacy on ACR $20 / 50 / 70$ criteria and DAS28 $<2.6$ but had similar efficacy versus csDMARDs on HAQ-DI. Efficacy of sarilumab $200 \mathrm{mg}$ was similar versus certolizumab, etanercept, tofacitinib and tocilizumab $8 \mathrm{mg} / \mathrm{kg}$ monotherapy across all efficacy outcomes. All safety outcomes appeared similar for sarilumab $200 \mathrm{mg}$ versus all comparator monotherapies.

\section{Scenario Analyses}

After excluding the studies SATORI, CHANGE and Etanercept 309, sarilumab $200 \mathrm{mg}$ monotherapy was found to be statistically superior to placebo and adalimumab, and comparable to certolizumab, etanercept, tocilizumab $8 \mathrm{mg} / \mathrm{kg}$ IV and tofacitinib monotherapies. For the scenario in which TNF- $\alpha$ inhibitors treatments were pooled together, sarilumab $200 \mathrm{mg}$ monotherapy was found to be statistically superior versus placebo, csDMARDs, TNF- $\alpha$ inhibitors and tofacitinib. Compared with tocilizumab $8 \mathrm{mg} / \mathrm{kg} \mathrm{IV}$, sarilumab efficacy was comparable.

\section{DISCUSSION}

Active comparator-controlled, randomized trials evaluating the comparative efficacy and safety of bDMARDs and tsDMARDs for the treatment of RA are few and limited to adalimumab as active comparator, including the MONARCH study of sarilumab compared with adalimumab [2, 20-24]. The objective of this study was to evaluate the comparative efficacy and safety of SC sarilumab $200 \mathrm{mg}$ monotherapy, administered $\mathrm{q} 2 \mathrm{w}$ versus other globally approved monotherapies for RA.

Consistent with the head-to-head trial of sarilumab versus adalimumab in MONARCH, the present NMA indicated that sarilumab $200 \mathrm{mg}$ monotherapy had superior efficacy versus adalimumab monotherapy on all outcomes. It was also found that sarilumab had superior efficacy versus tofacitinib monotherapy on ACR20 and versus csDMARDs on ACR
$20 / 50 / 70$ criteria and DAS28 $<2.6$. On other outcomes for these monotherapies and against all the other tsDMARD monotherapies, similar efficacy was observed. Rates of SI/SAE for sarilumab monotherapy were equivalent to those of all bDMARD, tsDMARD and CsDMARD monotherapies.

These results are consistent with previously published NMAs of the comparator bDMARD, tsDMARD and csDMARD monotherapies in RA, without sarilumab included in the networks [25-27]. In a 2015 meta-analysis by Buckley et al., it was found that patients receiving a TNF inhibitor or tofacitinib had greater ACR 20/50/ 70 responses than those receiving placebo [27]. While tocilizumab resulted in greater responses than TNF inhibitor, these were not statistically significant.

A more recent 2017 NMA of 28 studies comparing biologic monotherapy versus methotrexate, placebo or other biologic monotherapy found that all agents, except anakinra and infliximab, were superior to placebo [26]. Furthermore, etanercept and rituximab were superior to anakinra and tocilizumab was superior to adalimumab, anakinra, certolizumab, and golimumab. No differences were found between etanercept, tocilizumab and rituximab. A Cochrane review, updated in 2016, compared the efficacy and safety of biologic or tofacitinib monotherapy in patients who had failed traditional DMARDs [25]. The analysis of 46 RCTs found that overall, biologic therapy was superior to placebo and methotrexate/other DMARDs. TNF inhibitor and non-TNF inhibitor showed similar efficacy. However, anakinra and tofacitinib showed similar results for HAQ as placebo.

\section{Strengths and Limitations}

One limitation of the study was the variability in placebo response observed across the network; however, given the large number of active-controlled trials for the monotherapy csDMARD-IR population, it was feasible to address this situation by conducting OR-NMA $[15,28]$. 
In addition, some of the comparative studies of etanercept, certolizumab pegol and tofacitinib required 2 intermediate comparators rather than 1 , thus creating a greater degree of uncertainty.

The strengths of this NMA include the range of efficacy and safety outcomes which have been considered, providing a comprehensive view of the comparative efficacy and safety of sarilumab to inform clinical decision-making and the conduct of health technology assessments. The most robust networks, ACR20/50, used only one common comparator on all comparisons with sarilumab on these endpoints. The scenario analyses confirmed the results against the base case analysis, where comparisons were feasible.

The present indirect comparison was conducted following best practice guidelines and demonstrated that sarilumab SC $200 \mathrm{mg}$ monotherapy has superior efficacy compared with adalimumab, as well as csDMARDs alone, and comparable or better efficacy and similar safety compared with other bDMARDs and tsDMARDs in the csDMARD-IR patient populations. Compared with tocilizumab $8 \mathrm{mg} / \mathrm{kg}$ IV, sarilumab $200 \mathrm{mg}$ had similar efficacy and safety.

\section{CONCLUSION}

In csDMARD-IR patients, sarilumab $200 \mathrm{mg}$ monotherapy has superior efficacy and similar safety versus csDMARDs, superior efficacy and similar safety versus adalimumab, and similar efficacy and safety versus bDMARDs and tsDMARDs.

\section{ACKNOWLEDGEMENTS}

We thank the participants of the original studies included in this analysis. The authors would also like to thank Parexel for conducting the literature searches and analyses.

Funding. This study, the article processing charges and open access fee were sponsored by
Sanofi and Regeneron Pharmaceuticals, Inc. The sponsor was involved in the study design, collection, analysis and interpretation of data, as well as data checking of information provided in the manuscript. The authors received no honoraria related to the development of this publication. All authors had full access to all of the data in this study and take complete responsibility for the integrity of the data and accuracy of the data analysis.

Medical Writing and Editorial Assistance. Medical writing assistance and editorial support, under the direction of the authors, were provided by Gauri Saal, MA Economics, and Sinead Stewart, both of Prime (Knutsford, UK), funded by Sanofi/Regeneron Pharmaceuticals according to Good Publication Practice guidelines (http://www.ismpp.org/gpp3).

Authorship. All named authors meet the International Committee of Medical Journal Editors (ICMJE) criteria for authorship for this article, take responsibility for the integrity of the work as a whole, and have given their approval for this version to be published.

Disclosures. Ernest Choy has received research grants, and consultancy and speaker fees, from Amgen, Biogen, Bristol Myer Scripps, Boehringer Ingelheim, Celgene, Chugai Pharma, Eli Lilly, Janssen, Novimmune, Novartis, Pfizer, Regeneron Pharmaceuticals, Inc., Roche, R-Pharm, Sanofi, Tonix and UCB. ThiMinh-Thao Huynh is an employee of Sanofi and holds stock and/or stock options in the company. Laurence Pollissard is an employee of Sanofi and holds stock and/or stock options in the company. Hubert van Hoogstraten is an employee of Sanofi and holds stock and/or stock options in the company. Paulo Carita is an employee of Sanofi and holds stock and/or stock options in the company. Clare Proudfoot is a former employee and current stockholder of Sanofi, and current employee of Novartis. Chieh-I Chen is an employee of Regeneron Pharmaceuticals, Inc., and holds stock and/or stock options in the company. Andreas Kuznik is an employee of Regeneron Pharmaceuticals, Inc., and holds stock and/or stock options in 
the company. Erin Mangan is an employee of Regeneron Pharmaceuticals, Inc., and holds stock and/or stock options in the company. Nick Freemantle has received consulting fees from Sanofi, Novo Nordisk, Ipsen, Allergan, Takeda, Biogen, Abbvie and Lifecell.

Compliance with Ethics Guidelines. This article is based on previously conducted studies and does not contain any studies with human participants or animals performed by any of the authors.

Data Availability. The datasets generated during and/or analysed during the current study are not publicly available as the sponsoring companies do not publicly share datasets, but are available from the corresponding author on reasonable request.

Open Access. This article is distributed under the terms of the Creative Commons Attribution-NonCommercial 4.0 International License (http://creativecommons.org/licenses/ by-nc/4.0/), which permits any noncommercial use, distribution, and reproduction in any medium, provided you give appropriate credit to the original author(s) and the source, provide a link to the Creative Commons license, and indicate if changes were made.

\section{REFERENCES}

1. Genovese MC, Fleischmann R, Kivitz AJ, RellBakalarska M, Martincova R, Fiore S, et al. Sarilumab plus methotrexate in patients with active rheumatoid arthritis and inadequate response to methotrexate: results of a phase III study. Arthritis Rheumatol. 2015;67(6):1424-37.

2. Burmester GR, Lin Y, Patel R, van Adelsberg J, Mangan EK, Graham NM, et al. Efficacy and safety of sarilumab monotherapy versus adalimumab monotherapy for the treatment of patients with active rheumatoid arthritis (MONARCH): a randomised, double-blind, parallel-group phase III trial. Ann Rheum Dis. 2017;76(5):840-7.

3. Strand V, Kosinski M, Chen CI, Joseph G, RendasBaum R, Graham NM, et al. Sarilumab plus methotrexate improves patient-reported outcomes in patients with active rheumatoid arthritis and inadequate responses to methotrexate: results of a phase III trial. Arthritis Res Ther. 2016;18:198.

4. Fleischmann R, van Adelsberg J, Lin Y, CastelarPinheiro GD, Brzezicki J, Hrycaj P, et al. Sarilumab and nonbiologic disease-modifying antirheumatic drugs in patients with active rheumatoid arthritis and inadequate response or intolerance to tumor necrosis factor inhibitors. Arthritis Rheumatol. 2017;69(2):277-90.

5. Epstein D. The use of Comparative Effectiveness Research and Health Technology Assessment in European countries: current situation and prospects for the future. www.ugr.es/ davidepstein/HTA\% 20in\%20european\%20countries.docx. Accessed 27 Feb 2017.

6. Huynh T, Proudfoot C, Chen C, Pollissard L, Kuznik A, van Hoogstraten $\mathrm{H}$, et al. Network meta-analysis of the efficacy and safety of sarilumab monotherapy and combination therapy in rheumatoid arthritis patients with intolerance or inadequate response to disease-modifying antirheumatic drugs. J Manag Care Spec Pharm. 2017;23:S75.

7. Hutton B, Salanti G, Caldwell DM, Chaimani A, Schmid $\mathrm{CH}$, Cameron $\mathrm{C}$, et al. The PRISMA extension statement for reporting of systematic reviews incorporating network meta-analyses of health care interventions: checklist and explanations. Ann Intern Med. 2015;162(11):777-84.

8. National Institute for Health and Care Excellence. Guide to the methods of technology appraisal 2013. https://www.nice.org.uk/process/pmg9/resources/ guide-to-the-methods-of-technology-appraisal-2013pdf-2007975843781. Accessed 27 Feb 2017.

9. National Institute for Health and Care Excellence. Adalimumab, etanercept, infliximab, certolizumab pegol, golimumab, tocilizumab and abatacept for rheumatoid arthritis not previously treated with DMARDs or after conventional DMARDs only have failed. https://www.nice.org.uk/guidance/ta375/res ources/adalimumab-etanercept-infliximab-certolizu mab-pegol-golimumab-tocilizumab-and-abataceptfor-rheumatoid-arthritis-not-previously-treated-wi th-dmards-or-after-conventional-dmards-only-havefailed-82602790920133. Accessed 27 Feb 2017.

10. Jansen JP, Fleurence R, Devine B, Itzler R, Barrett A, Hawkins $\mathrm{N}$, et al. Interpreting indirect treatment comparisons and network meta-analysis for healthcare decision making: report of the ISPOR Task Force on Indirect Treatment Comparisons Good Research Practices: part 1. Value Health. 2011;14(4):417-28.

11. Hoaglin DC, Hawkins N, Jansen JP, Scott DA, Itzler $\mathrm{R}$, Cappelleri JC, et al. Conducting indirect-treatment-comparison and network-meta-analysis 
studies: report of the ISPOR Task Force on Indirect Treatment Comparisons Good Research Practices: part 2. Value Health. 2011;14(4):429-37.

12. Nuesch E, Trelle S, Reichenbach S, Rutjes AW, Tschannen B, Altman DG, et al. Small study effects in meta-analyses of osteoarthritis trials: meta-epidemiological study. BMJ. 2010;341:c3515.

13. Bucher HC, Guyatt GH, Griffith LE, Walter SD. The results of direct and indirect treatment comparisons in meta-analysis of randomized controlled trials. J Clin Epidemiol. 1997;50(6):683-91.

14. Lu G, Ades AE. Combination of direct and indirect evidence in mixed treatment comparisons. Stat Med. 2004;23(20):3105-24.

15. Dias S, Sutton AJ, Welton NJ, Ades AE. NICE DSU Technical Support Document 3: Heterogeneity: subgroups, meta-regression, bias and bias-adjustment. http://www.nicedsu.org.uk/TSD3\%20Hetero geneity.final\%20report.08.05.12.pdf. Accessed 27 Feb 2017.

16. Machado DA, Guzman RM, Xavier RM, Simon JA, Mele L, Pedersen R, et al. Open-label observation of addition of etanercept versus a conventional disease-modifying antirheumatic drug in subjects with active rheumatoid arthritis despite methotrexate therapy in the Latin American region. J Clin Rheumatol. 2014;20(1):25-33.

17. Caldwell DM, Ades AE, Higgins JP. Simultaneous comparison of multiple treatments: combining direct and indirect evidence. BMJ. 2005;331(7521): 897-900.

18. Spiegelhalter DJ, Best NG, Carlin BP, van der Linde A. Bayesian measures of model complexity and fit. J R Stat Soc B. 2002;64:583-639.

19. Fleischmann R, Cutolo M, Genovese MC, Lee EB, Kanik KS, Sadis S, et al. Phase IIb dose-ranging study of the oral JAK inhibitor tofacitinib (CP-690,550) or adalimumab monotherapy versus placebo in patients with active rheumatoid arthritis with an inadequate response to disease-modifying antirheumatic drugs. Arthritis Rheum. 2012;64(3): 617-29.

20. Weinblatt ME, Schiff $M$, Valente $R$, van der Heijde D, Citera G, Zhao C, et al. Head-to-head comparison of subcutaneous abatacept versus adalimumab for rheumatoid arthritis: findings of a phase IIIb, multinational, prospective, randomized study. Arthritis Rheum. 2013;65(1):28-38.

21. Gabay C, Emery P, van Vollenhoven R, Dikranian A, Alten R, Pavelka $\mathrm{K}$, et al. Tocilizumab monotherapy versus adalimumab monotherapy for treatment of rheumatoid arthritis (ADACTA): a randomised, double-blind, controlled phase 4 trial. Lancet. 2013;381(9877):1541-50.

22. Schiff $M$, Weinblatt ME, Valente $R$, van der Heijde D, Citera G, Elegbe A, et al. Head-to-head comparison of subcutaneous abatacept versus adalimumab for rheumatoid arthritis: two-year efficacy and safety findings from AMPLE trial. Ann Rheum Dis. 2014;73(1):86-94.

23. van Vollenhoven RF, Fleischmann R, Cohen S, Lee EB, Garcia Meijide JA, Wagner S, et al. Tofacitinib or adalimumab versus placebo in rheumatoid arthritis. N Engl J Med. 2012;367(6):508-19.

24. Taylor PC, Keystone EC, van der Heijde D, Weinblatt ME, Del Carmen Morales L, Reyes Gonzaga J, et al. Baricitinib versus placebo or adalimumab in rheumatoid arthritis. N Engl J Med. 2017;376(7):652-62.

25. Singh JA, Hossain A, Tanjong Ghogomu E, Mudano AS, Tugwell P, Wells GA. Biologic or tofacitinib monotherapy for rheumatoid arthritis in people with traditional disease-modifying anti-rheumatic drug (DMARD) failure: a cochrane systematic review and network meta-analysis (NMA). Cochrane Database Syst Rev 2016;11:Cd012437.

26. Tarp S, Furst DE, Dossing A, Ostergaard M, Lorenzen T, Hansen MS, et al. Defining the optimal biological monotherapy in rheumatoid arthritis: a systematic review and meta-analysis of randomised trials. Semin Arthritis Rheum. 2017;46(6):699-708.

27. Buckley F, Finckh A, Huizinga TW, Dejonckheere F, Jansen JP. Comparative efficacy of novel DMARDs as monotherapy and in combination with methotrexate in rheumatoid arthritis patients with inadequate response to conventional DMARDs: a network meta-analysis. J Manag Care Spec Pharm. 2015;21(5):409-23.

28. National Institute for Clinical and Economic Review. Targeted immune modulators for rheumatoid arthritis: effectiveness and value. Evidence report. https://icer-review.org/wp-content/uploads/ 2016/08/NE_CEPAC_RA_Evidence_Report_FINAL_ 040717.pdf. Accessed 7 Nov 2017. 